\title{
Rapport au savoir et pratiques des élèves: la notion de jeux alternatifs
}

\section{Patrice Rilhac}

Cet article présente les résultats d'une recherche dont l'ambition est de comparer les pratiques didactiques d'élèves d'une classe au niveau de l'enseignement secondaire obligatoire (élèves de 13 ans), confrontés à des enjeux de savoirs spécifiques: initiation à la démonstration en mathématiques et initiation à l'escalade en éducation physique et sportive. Notre approche méthodologique s'appuie sur la notion d'«algorithme méthodologique». Les résultats obtenus mettent en lumière l'existence de différentes formes de jeux alternatifs, nommés ainsi parce quills ne sont pas, stricto sensu, la forme de jeux épistémiques souhaités par les professeurs.

\section{Introduction}

Cet article se propose de présenter les résultats d'une recherche ${ }^{1}$ dont l'ambition était de rendre compte de pratiques d'élèves d'une classe de $4^{\mathrm{e}}$ de collège (13 ans), alors qu'ils étaient confrontés à des enjeux de savoirs différents en mathématiques lors d'une séquence d'«initiation à la démonstration» et en éducation physique et sportive lors d'une séquence d'«initiation à l'escalade» sur une structure artificielle d'escalade.

Après avoir présenté le cadre théorique et la méthodologie support des analyses menées, nous procéderons à une première mise en perspective des pratiques didactiques identifiées chez les élèves en classe de géométrie puis en classe d'escalade. Dans une seconde partie, nous comparerons ces pratiques. Nous montrerons alors l'existence de formes de jeux génériques. Nous les avons nommés jeux alternatifs (Rilhac, 2008) parce qu'ils ne sont pas, stricto sensu, la forme de jeux d'apprentissage à laquelle les enseignants souhaitaient voir jouer les élèves. Nous prolongerons cette dimension des jeux alternatifs en synthétisant certains des mécanismes spécifiques qui participent à l'altération, au sens non péjoratif de modification de l'état ou de la qualité d'une chose, des jeux d'apprentissage initialement prévus par les enseignants. 


\section{Analyse conceptuelle}

\section{Analyse de la pertinence}

En situation d'enseignement-apprentissage, la grande difficulté des élèves (Bautier \& Rayou, 2009; Charlot, 1997; Duru-Bellat \& Dubet, 2004; Hussenet \& Santana, 2004), au sens d'incapacité à répondre aux exigences du système éducatif, est perçue, par une part significative des professeurs de collèges, en termes de manques en mathématiques. En éducation physique et sportive, la grande difficulté apparaît sous les traits d'une inadéquation entre les capacités de l'élève et le système (Les Dossiers [MEN], 2007).

Si nous devons admettre que les dimensions sociales, psychologiques et cognitives constituent des axes de questionnement qui participent à la compréhension de la grande difficulté scolaire, nous ne devons pourtant pas oblitérer que les pratiques didactiques de ces élèves sont à considérer en lien avec des moments d'enseignement et d'apprentissage. Il faut, en effet, garder à l'esprit que tout acte d'enseignement et d'apprentissage relève de transactions didactiques (Sensevy, 2007) et que ces dernières sont en partie langagières.

Notre objectif premier vise à mettre en lumière les pratiques didactiques d'élèves d'une classe de $4^{\mathrm{e}}$, élèves majoritairement considérés, par leurs professeurs, comme étant en grandes difficultés d'apprentissage, mais toujours désireux de s'inscrire dans le cadre de la relation didactique (Mercier, 2008) avec l'enseignant.

Notre approche s'inscrit également dans une démarche comparatiste. Les pratiques d'élèves sont, le plus souvent, analysées en privilégiant un seul domaine disciplinaire. Dès lors, elles sont étudiées dans un seul contexte (Lahire, 1998). Plus rares, à notre connaissance, sont les études où l'activité des élèves en grande difficulté scolaire est envisagée à partir de domaines disciplinaires non connexes. Or, à l'école, les élèves sont confrontés à différentes matières.

Une telle approche est concevable dès lors que nous acceptons l'idée que toute pratique didactique peut être considérée comme une activité transactionnelle, parce qu'il est postulé que ce qui donne forme à ces transactions ce sont «leurs contenus, et que ces contenus sont des contenus de savoirs» (Sensevy, 2007, p. 17), au centre desquels les concepts de jeu (Chauvier, 2007) et d'institution (Douglas, 1999) sont essentiels.

\section{Cadre théorique}

\section{Enseigner et apprendre: un jeu didactique coopératif}

Enseigner consiste à transmettre un savoir afin qu'un élève, confronté à un problème (Joshua \& Dupin, 2003; Sensevy, 2007), parvienne à produire la bonne réponse. 
Dans cette logique, il incombe à l'enseignant de placer l'élève face à des situations-problème. L'objectif visé est d'amener l'élève à reconsidérer ses schèmes (Vergnaud \& Récopé, 2000) usuels, dès lors qu'ils font obstacle à une stratégie efficiente.

À titre d'exemple, lors d'une séquence d' «initiation à la démonstration", dans une classe de 4 e, pour démontrer l'existence mathématique d'une figure géométrique, il va s'agir de faire appel au raisonnement mathématique. "Démontrer c'est déduire de nouvelles propriétés à partir de propriétés connues et de règles de logiques» (Cahier de Mathématiques $4 \mathrm{e}^{2}$ )

Or, raisonner, dans la vie de tous les jours, c'est avant tout faire appel à la logique naturelle que "nous utilisons [...] dès lors que nous employons les expressions $\mathrm{Si}$... alors... ou il suffit que..., ou encore il est nécessaire que...» (DeloustalJorrand, 2004, p. 9).

Dans ce prolongement, enseigner c'est inhiber (Houdé, 2008) des modes opératoires qui constituent un obstacle à la construction de stratégies efficientes, que cela soit en mathématiques ou en éducation physique et sportive.

Dans cette perspective, l'activité (au sens de Pastré, 2004) du professeur et de l'élève peut être considérée comme un jeu coopératif conjoint (au sens de Sensevy, 2007), au sens où le professeur gagne dès lors que l'élève parvient à adapter le savoir à la situation rencontrée. Réciproquement, un élève gagne si son action (Durand, Saury \& Sève, 2006) répond aux attentes de savoir visé par l'enseignant.

Cependant, la pérennité du gain n'est réelle que si ce gain se reproduit dans le cadre de situations variables. Prenons deux exemples pour bien faire comprendre ce point essentiel.

En escalade, il ne suffit pas de disposer de techniques de grimpe comme, par exemple, savoir varier les saisies de prises (Testevuide, 2003). Savoir grimper consiste, en particulier, à organiser ses gestuelles aux contingences d'une voie, au fur et à mesure d'une ascension. Dès lors que l'élève parvient à adapter ses saisies au franchissement de différentes zones problématiques, il pourra être attesté de la conversion (telle que définie par Vergnaud, 1996) d'un savoir technique en un savoir pratique (tel que définie par Bourdieu, Chamboredon \& Passeron, 2005). Pour autant, ce savoir pratique n'évoluera en savoir théorique que si l'élève incorpore, au sens d'intégrer un élément afin qu'il fasse partie d'un tout seul à même de favoriser l'évolution d'une pratique en praxis, les dimensions biomécaniques, bioénergétiques et bio-affectives comme données inhérentes à la pratique de l'escalade.

En géométrie, lorsque nous nous intéressons à l'objet d'enseignement «initiation à la démonstration» en classe de $4^{\mathrm{e}}$, tout raisonnement doit être en lien avec les outils mathématiques présents dans l'énoncé. Cet énoncé peut prendre la forme d'une figure géométrique ou d'un texte écrit. En d'autres termes, un élève 
ne peut prouver l'existence mathématique d'un parallélogramme en se contentant d'observer une figure géométrique, ni en prenant des exemples. Ainsi, prouver qu'une figure est un parallélogramme nécessite de l'élève qu'il convoque les «outils» mathématiques utiles à la démonstration. Puis il devra organiser son argumentation sous la forme d'une écriture mathématique du type modus ponens.

Le modus ponens est un type de raisonnement logique consistant à affirmer une implication: si A, alors B. Le terme modus ponens vient du fait qu'on pose A afin de tirer la conclusion.

C'est alors seulement qu'il pourra être fait état d'une conversion d'un savoir théorique (les propriétés, les définitions, l'utilisation circonstanciée de symboles, etc.) en une praxis mathématique.

Enseigner et apprendre relèvent d'une activité asynchrone qui nécessite, pour le professeur, de coordonner son activité en fonction de l'action de l'élève. Inversement, l'élève aura également à adapter son activité en regard des situations présentées par le professeur.

Néanmoins, le professeur doit maintenir une certaine opacité quant à ses intentions didactiques, sous peine de vider de sa substance la relation didactique. Autrement dit, si un professeur, par un procédé quelconque, dévoile le savoir attendu - la propriété en géométrie, ou la gestuelle en escalade, l'élève produira, vraisemblablement, la bonne réponse, mais l'apprentissage risque d'être des plus aléatoires. En effet, il y aura une forte probabilité que, du côté de l'élève, la bonne propriété ou la gestuelle demandée, sera produite sans lien organique avec des enjeux épistémiques, mais pour satisfaire la demande de «bonne» réponse de l'enseignant. Dans ce cas, nous sommes loin d'une activité constructive (Pastré, 2004) rendue indispensable pour "traiter une classe de problèmes donnés» (Schubauer-Leoni, Leutenegger, Ligozat \& Flückiger, 2007, p. 54).

Par conséquent l'activité d'enseignement et d'apprentissage peut être envisagée comme une forme de jeu didactique institutionnel au sens où un professeur gagne si l'élève parvient à produire raisonnablement un savoir proprio-motu (Sensevy, 2007).

Dans cette perspective, nous pouvons percevoir qu'un élève doit satisfaire à un double jeu. Le premier consiste à répondre ici et maintenant à une demande spécifique du professeur en lien avec une situation rencontrée à un moment $t$. Le second jeu, que nous nommons jeu épistémique, nécessite que l'élève soit en capacité, selon les situations rencontrées, de convoquer les savoirs qui lui ont été enseignés, voire d'autres. Ainsi, pourront se construire de nouveaux savoirs à partir de connaissances émergeant des actions et rétroactions des milieux matériels - les outils supports à l'activité de l'élève et symboliques - les notions et concepts. 
Dans cette acception, une relation didactique sous-tend que l'élève développe une certaine intelligence des intentions professorales. Simultanément, il importe que le professeur développe une certaine intelligence des attentes des élèves (Mercier, 2008). Aussi, la notion de contrat didactique (Brousseau, 1998, 2003), en tant que système d'attentes réciproques dans un milieu donné, constitue une partie inhérente à toute tentative visant à la compréhension de pratiques d'enseignement et d'apprentissage.

Ainsi, pour comparer des pratiques didactiques in situ d'élèves en grandes difficultés, il nous apparaît pertinent d'inscrire nos analyses en nous appuyant sur la notion de jeux d'apprentissage (Sensevy, 2007; Sensevy, Mercier, Schubauer-Leoni, Ligozat \& Perrot, 2005 ), dans le cadre d'une dialectique milieuxcontrats.

Cette proposition nous la modélisons sous la forme suivante:

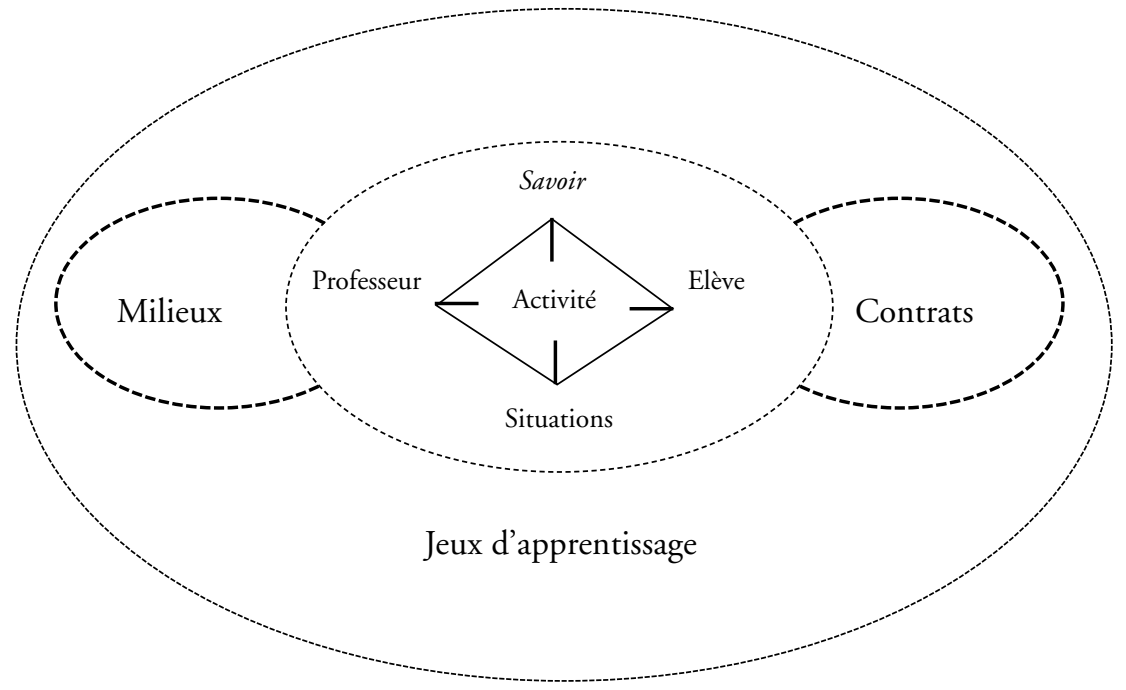

\section{Figure 1}

Les notions présentées ne sauraient suffire à rendre compte de pratiques des élèves. Nous avons donc fait appel à des notions fonctionnelles éprouvées en didactique de l'éducation physique et sportive, des mathématiques et en didactique comparée.

Les notions de chronogénèse, de topogénèse et de mésogénèse (Amade-Escot, 2003; Chevallard, 1991; Sensevy, 2007; Sensevy, Mercier \& Schubauer-Leoni, 2000) ont été convoquées à cette fin. 


\section{Mésogénèse, chronogénèse et topogénèse et agir du professeur}

Le professeur, dans le cadre d'un objet d'étude, organise le milieu (mésogénèse) par le biais de situations. Pour cela, il va disposer d'outils de savoirs matériels et symboliques afin que son enseignement puisse se traduire en apprentissage par les élèves. Ce choix est organisé par les actions attendues puis produites par les élèves.

Tout professeur est également le gardien du temps didactique (chronogénèse). C'est, en effet, lui qui détermine à quel moment d'une situation, d'une leçon, un savoir devra apparaître ou réapparaître.

Enfin, c'est aussi lui qui détermine ce qui revient à la charge de l'élève. Par le biais de situations et d'actes de langage, compris au sens de signes verbaux ou gestuels, il détermine la nature des responsabilités vis-à-vis des savoirs. En d'autres termes, il définit la position (topogénèse), vis-à-vis du savoir enseigné, que l'élève doit occuper à chaque instant (chronogénèse) et selon chaque milieu (mésogénèse).

Néanmoins, un enseignant ne peut agir si l'élève n'adhère pas au jeu didactique. C'est pourquoi l'activité de l'enseignant est, certes, dépendante de la relation didactique, mais aussi elle nécessite le maintien de cette relation tout au long de la séquence d'enseignement.

\section{Mésogénèse, chronogénèse et topogénèse et agi $r$ de l'élève}

Si un élève n'est pas sans connaissances, il est pourtant un ignorant parce qu'il n'a pas pleinement conscience du jeu épistémique visé par l'enseignant. Un jeu objectivé par des situations qui lui permettront de s'accommoder aux situations-problèmes rencontrées lors d'une évaluation sommative.

C'est pourquoi le jeu épistémique du professeur n'est accessible à un élève que progressivement, au fur et à mesure de l'avancée du temps des savoirs (chronogénèse), à partir de situations (mésogénèse) et selon la place topogénétique que lui accorde l'enseignant.

Notre approche des pratiques des élèves en grandes difficultés d'apprentissage se veut clinique au sens où nous cherchons d'abord à rendre compte, afin de mieux les comprendre, des phénomènes didactiques du type incidents didactiques. Ils sont nommés ainsi parce qu'ils ne correspondent pas à l'attente de savoir visé par l'enseignant, sans pour autant que ces hiatus soient nécessairement compris des enseignants.

Le processus enseigner-apprendre, du point de vue des relations qui s'établis- 
sent entre professeur et élèves et de leur(s) rapport(s) au(x) savoir(s), est abordé en référence à une théorie anthropologique 3 en didactique. Elle a pour objectif de mieux comprendre le fonctionnement du contrat didactique (Amade-Escot \& O'Sullivan, 2007: Brousseau, 1998).

À partir de l'étude d'interactions didactiques, in situ, entre des élèves d'une même classe confrontés à des enjeux de savoirs spécifiques, nous pensons lutter contre «la cécité à l'aspect», l'inaptitude à saisir ou remarquer l'apparition [...]» (Chauviré \& Sackur, 2003, p. 11) de formes de jeux inappropriés à l'acquisition de savoirs.

\section{Synthèse des connaissances}

Cette recherche a été engagée parce qu'il n'existait pas, à notre connaissance, de travaux comparatistes consacrés à l'observation de pratiques didactiques d'élèves en grandes difficultés appartenant à une même classe, alors qu'ils évoluaient au sein de disciplines aux enjeux de savoirs spécifiques.

D'autres recherches se sont intéressées à mieux comprendre les comportements d'élèves en situations de difficultés d'apprentissage à partir de dimensions micro-sociologiques (Charlot, 2003), psychanalytiques (Blanchard-Laville, 2002), psychologiques (Laterasse \& Brossais, 2006). Elles constituent autant d'entrées nécessaires à la compréhension des pratiques didactiques d'élèves en difficulté.

Toutefois, la dimension anthropologique en didactique, autrement dit la relation construite par un élève en grande difficulté dans le cadre d'une relation didactique acceptée, est au moins aussi dépendante du désir d'être reconnu en tant que sujet «viable» de l'institution classe.

\section{Méthodologie}

L'analyse de pratiques didactiques en situations de classes ordinaires suppose de procéder à la collecte de l'ensemble des données nécessaires à l'analyse d'interactions de sujets et des artéfacts qu'ils utilisent.

La première des difficultés, après avoir pu procéder à l'organisation des conditions techniques de collecte des données, est de parvenir à l'identification de moments didactiques parmi un ensemble d'autres faits. Quels procédés seraient susceptibles de nous aider à cerner des faits didactiques pertinents pour notre étude? Comment agir pour que les faits retenus pour l'étude ne le soient pas parce qu'ils sont déjà entachés de sens conformes aux fins poursuivies (Durkheim, 2002)?

Pour répondre à ce questionnement, l'approche de chacune des séances a d'abord fait l'objet de nombreuses lectures en continu, à partir des enregistre- 
ments audio et vidéo et des transcrits, afin de nous imprégner des scènes d'énonciation.

«En parlant de scène d'énonciation on met l'accent sur le fait que l'énonciation advient dans un espace institué, défini par le genre du discours, mais aussi par la dimension constructive du discours, qui se met en scène, instaure son propre espace d'énonciation» (Charaudeau \& Maingueneau, 2002, p. 515).

Avant d'aborder ces lectures en continu, nous avions procédé à un entretien non directif de chacun des professeurs. Ces entretiens, ante séquences, visaient à dégager les principaux enjeux de savoirs (Schubauer-Leoni \& Leutenegger, 2002).

Nous n'avons pas conduit d'entretiens ante séances. Nous ne voulions pas prendre le risque d'influencer les façons de faire habituelles des professeurs et des élèves.

Ce risque était d'autant plus réel que nous avions décliné l'objet de la recherche. Nous avions en particulier précisé que notre intérêt portait sur les savoirs appris par les élèves au fur et à mesure de l'avancée du temps d'enseignement.

Les lectures en continu, précédées d'un entretien ante séquence, associées à un travail épistémique, nous ont permis de percevoir la trame didactique des séquences d'enseignement.

\section{Environnement}

\section{Les élèves}

Dans le cadre de notre objet d'étude, notre choix s'est porté sur les élèves d'une classe de $4^{\mathrm{e}}$ de collège composée de 12 filles et de 12 garçons, parce que:

- Ces élèves étaient considérés, dans leur grande majorité, en grande difficulté d'apprentissage depuis l'école primaire. Cette difficulté d'apprentissage avait été à nouveau mise en évidence lors d'évaluations du début de l'année de $6 \mathrm{e}$. Elle n'avait fait que se renforcer depuis.

- Ces élèves, malgré leur grande difficulté scolaire, étaient toujours désireux de progresser.

- Nous connaissions la plupart des élèves, dans le cadre d'un enseignement les deux années précédentes. Il nous était ainsi plus aisé d'entrer en relation avec eux dès lors que nous aurions à les solliciter en regard de choix de savoirs convoqués.

\section{Le choix des élèves}

Au plan de la spécificité des disciplines abordées, le choix des élèves a été guidé par les critères suivants:

- En géométrie, l'élève devait s'inscrire dans le cadre de la relation didactique. Pour cela, il fallait qu'il accepte de répondre de sa propre initiative aux questions de l'enseignante. De plus, ces réponses devaient être organisées à partir de savoirs liés à l'objet d'enseignement. 
- En escalade, l'élève devait également montrer son adhésion aux situations proposées par l'enseignant. Parallèlement, son activité de grimpe ne devait pas être entravée par une sensation de vertige $v r a i^{4}$.

Au plan générique:

- La situation didactique dans laquelle évoluait l'élève devait pouvoir être identifiée.

- Les pratiques de l'élève devaient être porteuses de traces (Ginzburg, 1989) didactiques potentiellement en décalage avec le jeu épistémique sous-jacent.

\section{Les activités d'enseignement}

Le choix des objets d'enseignement «initiation à la démonstration» et «initiation à l'escalade» fut déterminé parce que nous pouvions appuyer nos analyses a priori en nous référant à des travaux de recherches existants ou, au moins, à des études déjà menées par des experts de chacune des disciplines.

La question de la taille de l'objet d'étude a participé à la détermination des objets d'enseignements retenus. Un nombre de leçons relativement limité en escalade et en géométrie, cinq leçons dans chacune des disciplines, associé au fait que ces deux objets d'enseignement nous permettaient d'enregistrer l'ensemble des interactions entre professeurs-élèves et élève(s)-élève(s), a fini par affirmer notre choix.

Enfin, notre attention centrée sur des activités en géométrie et en escalade est due à l'introduction d'un nouveau savoir, tout du moins du point de vue des professeurs.

\section{Instrumentation}

Chacune des cinq leçons a fait l'objet d'un enregistrement vidéo et audio dans l'ordre chronologique de leur déroulement.

Le dispositif pour recueillir les données était composé de deux caméras vidéo. La première caméra était axée sur les élèves, la seconde sur l'enseignant. Du côté de la prise de son, nous avons placé quatre micros d'ambiance pour pouvoir enregistrer les échanges des élèves. Le professeur était muni d'un micro HF. L'ensemble du matériel technique était relié à une table de mixage portative.

\section{Déroulement des observations}

En classe de géométrie, les enregistrements ont été effectués sans que soit présent le chercheur. Il s'agissait de perturber le moins possible le déroulement des séances. En escalade, la présence du chercheur a été rendue indispensable afin de pouvoir suivre les évolutions des élèves 5 . 
Nous avons enregistré l'intégralité des cinq leçons. Puis, dans un second temps, nous avons transcrit la totalité des interactions pour chacune des cinq leçons à partir des données recueillies.

\section{Méthode d'analyse des résultats}

L'analyse des pratiques des élèves a été abordée à partir de la notion d'algorithme méthodologique (Sensevy, à paraître). Cette approche nous a semblé pertinente dès lors qu'elle est envisagée, dans un premier mouvement, sur le strict plan du savoir disciplinaire visé.

\section{Éléments de méthode}

L'intérêt porté à l'étude comparative de pratiques didactiques d'élèves en grandes difficultés in situ nécessite la mise en œuvre d'une méthodologie adaptée. L'une des difficultés principales est de ne pas oblitérer les spécificités individuelles et intradisciplinaires des pratiques didactiques, tout en rendant accessibles certains des traits génériques de ces pratiques.

Dans ce dessein, des entretiens ante séquences des deux enseignants ont été conduits afin de définir les savoirs visés. Ces entretiens furent prolongés par un travail épistémique, lequel a été mené en lien avec des études déjà opérées dans chacune des disciplines. Nous avons ainsi pu préciser les enjeux de savoirs sousjacents visés lors des différents moments d'une leçon.

Dans un troisième temps, la transcription de l'intégralité des leçons a été opérée dans l'ordre chronologique pour chacune d'elles. Enfin, à partir de la notion de synopsis (Sensevy, Schubauer-Leoni \& Mercier, 2007), nous avons pu opérer l'étude micro didactique des pratiques didactiques des élèves retenus.

\section{Notion de synopsis}

Un synopsis est un résumé du scénario d'une leçon afin d'en écrire l'histoire, telle qu'elle s'est produite et non telle que l'on pense qu'elle s'est produite au seul visionnement d'une vidéo. Il s'agit, pour le chercheur, de s'imprégner du contexte dans lequel évoluent les acteurs.

Le synopsis d'une leçon peut être découpé en acte(s), scène(s) et en épisode(s).

- Un acte correspond à la subdivision d'une leçon. Il se caractérise par une certaine unité de temps et de lieu. Ces actes peuvent être divisés en scènes.

- Une scène représente une partie de la leçon. La scène permet de cerner le tempo d'une leçon, au sens d'allure particulière qu'un professeur donne au déroulement de ses actions didactiques.

Pour avoir accès à l'histoire des savoirs d'une leçon, nous avons eu recours à la technique de la paraphrase ${ }^{6}$.

Par le découpage d'une leçon en actes/scènes et épisodes et la technique de la paraphrase, il nous a été possible de reconstituer l'intrigue didactique de chacune 
des classes, leçons après leçons. Nous avons ainsi pu mettre en relief des évènements didactiques au sein desquels sont apparus des incidents didactiques (Amade-Escot \& Marsenach, 1995).

Nous entendons par événement un fait qui survient à un moment donné. Il se caractérise par une transition, voire une rupture, dans le cours des choses. Dans le domaine didactique, l'intérêt se porte préférentiellement sur les temps d'introduction (ou réintroduction) d'objet(s) convoqués par les acteurs lors de phases de transactions.

Nous avons conduit notre cheminement méthodologique de manière similaire dans les deux disciplines, à cette nuance près qu'en escalade les interactions professeur-élève(s), apparaissent, le plus souvent, du côté des élèves, sous la forme de gestuelles. Nous avons donc eu recours à l'utilisation de photogrammes (Forest, 2006; Rilhac, 2008).

Un photogramme permet de transcrire un langage corporel, lequel nous permet de segmenter précisément les gestuelles d'un grimpeur.

L'objectif recherché est d'opérer une digitalisation d'un acte de langage (Austin, 1970) de nature analogique, puisque non verbale, afin de rendre visibles les savoirs mis en jeu par l'élève.

Notre pouvons schématiser notre approche méthodologique sous la forme suivante:

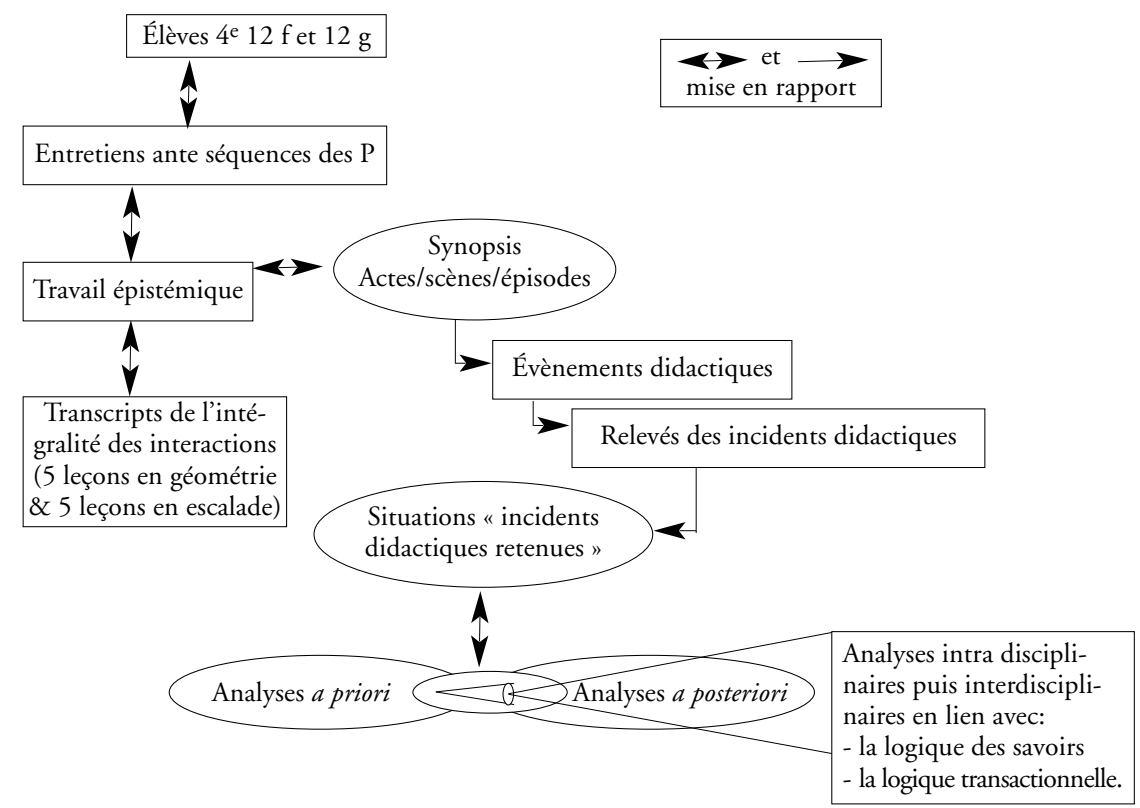

Figure 2: Algorithme méthodologique pour une approche des pratiques didactiques en situations de classes ordinaires d'escalade et de géométrie 
Les analyses opérées à partir de cette approche nous ont permis, dans un premier temps, de relever les usages problématiques rencontrés par les élèves.

\section{Résultats}

Les premiers résultats des analyses menées lors des cinq séances, «initiation à la démonstration» en géométrie et «initiation à l'escalade» en éducation physique et sportive, laissent transparaître les formes de pratiques suivantes des élèves.

Pour une lecture plus compréhensive du tableau récapitulatif des usages problématiques rencontrés par les élèves, présenté ci-dessous, la notion de praxis est à envisager en termes d'activité didactique attendue de l'élève par le professeur. Dans cette acception, une praxis souligne l'enjeu épistémique sous-jacent au jeu de savoir(s) proposé aux élèves aux moments $t$ dans le cadre de situations d'enseignement-apprentissage.

La notion d'usages problématiques est à considérer en lien avec l'action effective déployée par les élèves en regard des situations présentées par le professeur.

\section{Premiers résultats: usages problématiques des pratiques développées par les élèves en escalade et en géométrie}

\section{Tableau 1: Usages problématiques rencontrés par les élèves en escalade et en géométrie}

\begin{tabular}{|c|c|c|c|}
\hline \multicolumn{2}{|c|}{ Escalade } & \multicolumn{2}{|c|}{ Géométrie } \\
\hline Praxis & Usage problématique & Praxis & Usage problématique \\
\hline $\begin{array}{l}\text { Identifier les passages } \\
\text { d'une voie pour antici- } \\
\text { per les déplacements }\end{array}$ & $\begin{array}{l}\text { Production de gestuelles } \\
\text { usuelles. Lecture au } \\
\text { "coup par coup» au fur } \\
\text { et à mesure de la } \\
\text { progression }\end{array}$ & $\begin{array}{l}\text { Lire un énoncé/figure } \\
\text { pour pouvoir utiliser les } \\
\text { propriétés et les } \\
\text { définitions }\end{array}$ & $\begin{array}{l}\text { Production de savoirs } \\
\text { déclaratifs }\end{array}$ \\
\hline $\begin{array}{l}\text { Produire des gestes dy- } \\
\text { namiques }\end{array}$ & $\begin{array}{l}\text { Non identification des } \\
\text { enjeux de savoirs }\end{array}$ & $\begin{array}{l}\text { Produire un raisonne- } \\
\text { ment mathématique à } \\
\text { partir des signes géomé- } \\
\text { triques adéquats }\end{array}$ & $\begin{array}{l}\text { Non identification de } \\
\text { l'enjeu de savoir }\end{array}$ \\
\hline $\begin{array}{l}\text { Produire des allers-re- } \\
\text { tours entre les conduites } \\
\text { motrices produites et les } \\
\text { contingences d'une voie }\end{array}$ & $\begin{array}{c}\text { Assujettissement aux } \\
\text { gestuelles préconisées } \\
\text { par l'enseignant }\end{array}$ & $\begin{array}{l}\text { Produire des allers-re- } \\
\text { tours entre des signes } \\
\text { mathématiques d'un } \\
\text { énoncé, d'une figure, et } \\
\text { la propriété choisie }\end{array}$ & $\begin{array}{l}\text { Assujettissement aux } \\
\text { règles procédurales édic- } \\
\text { tées par l'enseignant }\end{array}$ \\
\hline $\begin{array}{l}\text { Construire ses déplace- } \\
\text { ments en lien avec les } \\
\text { exigences d'un milieu } \\
\text { matériel }\end{array}$ & $\begin{array}{l}\text { Lecture triviale des } \\
\text { termes du contrat } \\
\text { didactique }\end{array}$ & $\begin{array}{l}\text { Construire une familia- } \\
\text { rité avec le jeu de lan- } \\
\text { gage logique de } \\
\text { l'implication }\end{array}$ & $\begin{array}{l}\text { Lecture triviale des } \\
\text { termes du contrat } \\
\text { didactique }\end{array}$ \\
\hline
\end{tabular}




\begin{tabular}{|c|c|c|c|}
\hline $\begin{array}{c}\text { Construire un discours } \\
\text { de savoir justificatif, } \\
\text { voire explicatifs des } \\
\text { techniques }\end{array}$ & $\begin{array}{c}\text { Lecture des situations } \\
\text { envisagées pour elles- } \\
\text { mêmes, indépendam- } \\
\text { ment les unes des autres. } \\
\text { La forme déclarative } \\
\text { des savoirs prévaut } \\
\text { sur la forme opératoire }\end{array}$ & $\begin{array}{c}\text { Construire une mise en } \\
\text { relation entre artefacts et } \\
\text { savoirs justificatifs }\end{array}$ & $\begin{array}{l}\text { Lecture des situations } \\
\text { encapsulées à des formes } \\
\text { instituées de savoirs, et } \\
\text { centrée sur les ostensifs } \\
\text { mathématiques manipu- } \\
\text { lés par l'enseignant. } \\
\text { La forme déclarative } \\
\text { des savoirs prévaut sur }\end{array}$ \\
\hline
\end{tabular}

Dans un second temps, une analyse comparative, interdisciplinaire, nous a permis de mettre en évidence un phénomène d'altération du jeu didactique. Nous

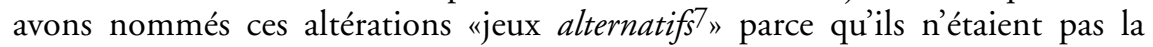
forme de jeux épistémiques à laquelle les professeurs souhaitaient voir jouer les élèves.

\section{Seconds résultats. Les pratiques des élèves: des jeux alternatifs}

\section{Notion de jeux alternatifs en géométrie}

L'engagement professoral à faire évoluer les jeux de langages naturels des élèves (logique naturelle) en jeu de langage mathématique, se trouve en rupture avec les règles qui contribuent à structurer l'activité des élèves. Pour ces derniers, il s'agit moins d'élaborer un mode de raisonnement logique mathématique que de donner à voir au professeur des signes d'illusion d'une activité mathématique. Mais pour que cela soit possible, professeur et élèves doivent co-construire un environnement dans lequel certaines formes de jeux doivent exister. Nous avons pu relever les formes de jeux suivants:

- Le jeu de participation. Participer au jeu signifie montrer son adhésion au jeu professoral en produisant des réponses allusives en lien avec ce que le professeur demande.

\begin{tabular}{|c|c|l|}
\hline $\begin{array}{c}\text { Tours de } \\
\text { parole }\end{array}$ & Locuteur & \multicolumn{1}{c|}{ Actes de langage } \\
\hline 54 & Professeur & $\begin{array}{l}\text { Alors quelle propriété je vais choisir là-dedans pour démontrer, pour prouver } \\
\text { que cette figure est bien un parallélogramme? Corinne. }\end{array}$ \\
\hline 55 & Corinne & Euh....la deuxième. \\
\hline
\end{tabular}

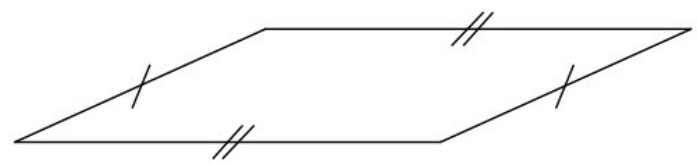

Mais il s'agit aussi, du côté du professeur, de maintenir la continuité de la relation en désignant les signes mathématiques utiles.

\begin{tabular}{l|l|l}
56 & Professeur & La deuxième! Je sais que les côtés sont parallèles deux à deux?
\end{tabular} 
Ainsi l'élève, en proposant une réponse évasive ou incomplète, et le professeur, en restreignant l'incertitude du milieu, agissent de concert pour que puisse exister le jeu de participation.

- Le jeu d'ostension des savoirs. Le jeu d'ostension des savoirs consiste à faire étalage de savoirs déclaratifs. Il peut s'agir de définitions, de propriétés, ou encore des mots mathématiques comme parallèles etc. L'extrait présenté ci-dessous permet de rendre compte de cette forme de jeu.

\begin{tabular}{|l|l|l|}
\hline 82 & Professeur & (...) Et si je dessine ça..., est ce que j’ai dessiné un parallélogramme? \\
\hline
\end{tabular}

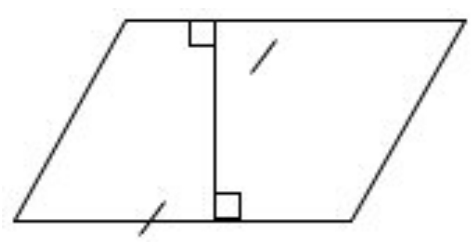

\begin{tabular}{|c|c|c|}
\hline 83 & élève & Non, c'est pas le même... \\
\hline 84 & $\begin{array}{l}\text { élèves } 1 \text {, } \\
2,3,4\end{array}$ & Si, non, si, si. \\
\hline 85 & Professeur & $\begin{array}{l}\text { Alors, qu'est ce que j'ai dit. Qu'est ce que mes symboles veulent dire? Qu'est } \\
\text { ce que ça veut dire ça? }\end{array}$ \\
\hline 86 & $\begin{array}{l}\text { Maxime + } \\
1,2,3\end{array}$ & Même longueur. \\
\hline 87 & $\mathrm{P}$ & Même longueur. Qu'est-ce que celle-là celle là? \\
\hline 88 & e 1,2 & Parallèle, la hauteur. \\
\hline 89 & $\mathrm{P}$ & Ces deux-là, elles sont? \\
\hline 90 & $\begin{array}{l}\text { Maxime }+ \\
1,2\end{array}$ & Parallèles \\
\hline 91 & $\mathrm{P}$ & $\begin{array}{l}\text { Parallèles. Alors, est-ce que c'est un parallélogramme, et si oui quelle } \\
\text { propriété j'utilise? }\end{array}$ \\
\hline 92 & Pierre & Euh...la troisième. \\
\hline 93 & $\mathrm{P}$ & $\begin{array}{l}\text { La troisième parce que j'ai deux côtés qui sont parallèles et qui ont la même } \\
\text { longueur. Je suis donc dans ce cas-là. Sur ces deux côtés je sais rien. Je sais as- } \\
\text { sez de choses sur ces deux côtés là. Je sais assez de choses pour dire que c'est un } \\
\text { parallélogramme (...). }\end{array}$ \\
\hline
\end{tabular}

Ainsi est exposée par les élèves une certaine connaissance mathématique. Des connaissances bien loin d'être incorporées à une praxis mathématique. Ce qui est mis en valeur, artificiellement, c'est l'intérêt pour l'objet enseigné. En retour cela permet, du côté du professeur, d'atténuer le sentiment diffus de ne pas parvenir 
à faire évoluer des schèmes usuels en opérations spécifiques pour traiter une classe de problèmes.

- Le jeu de réciprocité. Le jeu de réciprocité consiste en un échange de bons procédés. Dit autrement, les élèves ne s'engagent à échanger avec le professeur que si, en retour, ce dernier simplifie la situation au point que l'élève n'ait plus qu'à agir en opérateur d'algorithme (Pascal, 1980). Dès lors, en cas d'échec, l'élève attribuera préférentiellement sa non-réussite à l'enseignant. En représailles, l'enseignant pourra recevoir pour seule réponse à des questionnements ultérieurs un mutisme sélectif, voire une réponse aberrante. Il semble bien que ces types de comportements transcendent les disciplines, les cultures, les nationalités (Woods, 1990).

- Le jeu de conformité. Ce jeu sous-tend, du côté des élèves, que seul le professeur est dépositaire de la «bonne» stratégie pour parvenir aux «bonnes» réponses. Aussi à chaque fois qu'une réponse proposée n'est pas la bonne, c'est, pour l'élève, que les règles édictées par l'enseignant ne sont pas opératoires. Dans ce prolongement, les élèves peuvent attribuer leurs erreurs au fait que le professeur n'agit pas pour les aider. La confusion vient du fait que, pour ces élèves, apprendre à l'école est synonyme de déclamer des savoirs. Ainsi la connaissance est de nature déclarative, alors que pour l'enseignant la connaissance est opératoire (Pastré, 2004).

A contrario, du côté de l'enseignant, sa pratique consiste à exposer les règles incontournables à la résolution des énoncés. Dès lors, sa responsabilité ne saurait être totalement engagée puisque, à son sens, la règle utile pour parvenir à identifier la bonne propriété a été dévoilée.

\begin{tabular}{|c|l|}
\hline \multirow{3}{*}{ Professeur } & $\begin{array}{l}\text { Les données c'est ce qu'on sait, une propriété et une conclusion. La propriété on a le } \\
\text { droit de l'utiliser que si ce qui est ici (le professeur souligne au tableau) est la même chose } \\
\text { que ce qui est là. D'accord? La propriété j'ai le droit de l'utiliser que si j'ai entre le si et } \\
\text { le alors exactement la même chose que ce que j'ai au-dessus, en données. Et ma conclu- } \\
\text { sion, ma conclusion ça va être exactement ce qu'il y a après le alors. (le professeur sou- } \\
\text { ligne à nouveau) D'accord? } \\
\text { Donc quand on va choisir une propriété, on va faire en sorte que ce qui est en bleu soit } \\
\text { la même chose, et ce qui est en vert soit la même chose (le professeur entoure le mot don- } \\
\text { nées et l'espace vide entre si et alors). }\end{array}$ \\
\hline
\end{tabular}

\section{Notion de jeux alternatifs en escalade}

Pour les élèves observés, il apparaît également qu'il s'agit moins de construire les règles stratégiques qui permettraient de gravir une voie le plus économiquement possible, que de développer des formes corporelles en adéquation avec la demande supposée du professeur. Dans cette perspective, les formes de jeux de participation, d'ostension des savoirs, de réciprocité et de conformité, à vocation générique, n’en demeurent pas moins spécifiques: 
- Le jeu de participation, où s'engager physiquement, mais aussi solliciter d'autres situations de grimpe, est sans nul doute considéré comme une des attitudes à adopter pour espérer être reconnu en tant que «bon sujet» de l'institution classe d'escalade.

\begin{tabular}{|c|c|l|}
\hline $\begin{array}{c}\text { Tours de } \\
\text { parole }\end{array}$ & Locuteur & \multicolumn{1}{c|}{ Actes de langage } \\
\hline 18 & Nadia & Eh Monsieur!....Une fois qu'on a fait les trois fois*** \\
\hline 19 & Professeur & $\begin{array}{l}\text { Alors euh.... on change peut-être pas tout de suite (sous-entendu de voie). Tu } \\
\text { vas monter en essayant de ne pas marquer de temps d'arrêt sur cette petite } \\
\text { voie. }\end{array}$ \\
\hline
\end{tabular}

Une reconnaissance indispensable pour qui souhaite obtenir la meilleure note possible de participation, comme nous l'avons relevé sur la fiche d'évaluation du professeur. Tout comme cela est nécessaire à celui qui veut bénéficier d'une aide professorale, et ainsi devenir le détenteur d'une information primordiale sur les mouvements souhaités par l'enseignant comme nous l'indique l'extrait ci-dessous lors de la séance 4.

\begin{tabular}{|c|c|c|c|}
\hline \multirow{2}{*}{ Leçon 4} & $\begin{array}{c}\text { Tours de } \\
\text { parole }\end{array}$ & Locuteur & Actes de langage \\
\cline { 2 - 4 } & 18 & Professeur & $\begin{array}{l}\text { Il faut que tu utilises moins la force de tes bras que la force de tes } \\
\text { jambes. (...). Donc le but du jeu, c'est de bouger les pieds pour } \\
\text { avoir plus de facilité pour monter les mains. }\end{array}$ \\
\hline
\end{tabular}

- Le jeu d'ostension des savoirs. Dans le jeu d'ostension des savoirs, pour l'élève, il ne s'agit pas de déclamer des savoirs théoriques comme en géométrie, mais des savoirs pratiques. Par exemple, en grimpant en évitant de placer les mains et les pieds sur un même niveau de prises, et cela uniquement lorsque l'enseignant observe lors d'une situation où la forme de grimpe demandée est d'éviter de poser les mains ou les pieds sur un même niveau ${ }^{9}$ de prises.

Cette forme de pratique permet en retour, du côté du professeur, de se conforter dans le fait que son enseignement répond à la fois aux contenus qu'il visait, et aux attentes d'instruction voulue par l'Institution.

- Le jeu de réciprocité. Pour que le jeu de réciprocité puisse fonctionner, cela nécessite, en tout premier lieu, que l'élève fasse reconnaître son engagement physique comme signe d'adhésion forte au jeu de l'enseignant. Dès lors, si le professeur admet cet engagement comme signe d'adhésion à son jeu, il aura une certaine inclination à relever préférentiellement certains des aspects de la motricité de l'élève, ceux qui correspondent aux enjeux épistémiques sous-jacents. Cette approche professorale est similaire à l'effet Jourdain mis en avant par Guy Brousseau en mathématiques (Brousseau, 1998). Ainsi, le don d'engagement de l'élève est suivi d'un contre-don professoral (Mauss, 2001). 
- Le jeu de conformité consiste à appliquer le plus fidèlement possible les gestuelles demandées par le professeur selon les situations d'apprentissage, qu'il s'agisse de grimper en réduisant le nombre de prises utilisées, ou qu'il s'agisse de gravir la voie en plus de prises pieds que de prises mains. Or, du côté du professeur, si un élève ne réussit pas, sa responsabilité ne saurait être engagée, parce qu'il considérera avoir créé les conditions nécessaires de la réussite, en laissant à l'élève 1) le choix de la voie à gravir, 2) le libre arbitre quant au nombre de prises à utiliser. En conséquence si l'élève ne réussit pas cela ne peut être dû, au sens du professeur, qu'à l'absence d'engagement.

\begin{tabular}{|c|c|c|l|}
\hline & $\begin{array}{c}\text { Tours de } \\
\text { parole }\end{array}$ & Locuteur & \multicolumn{1}{c|}{ Actes de langage } \\
\cline { 2 - 4 } Leçon 2 & 9 & Professeur & $\begin{array}{l}\text { Aujourd'hui, proposition pour les débutants. La première des } \\
\text { conditions c'est de vouloir débuter, c'est de vouloir démarrer. } \\
\text { Donc pour démarrer, on peut travailler sur des positions de base } \\
\text { en traversée. [...] Voilà des idées, au moins pour démarrer. Si } \\
\text { vous ne démarrez pas, je ne peux pas prendre un fusil, un fouet } \\
\text { non plus, et puis vas-y, tu montes, tu montes. Et bien si tu ne } \\
\text { montes pas, tu ne montes pas. }\end{array}$ \\
\hline
\end{tabular}

Cette mise en perspective spécifique de la notion de jeux alternatifs en géométrie et en escalade devrait être approfondie. Il nous apparaissait important d'identifier certains des mécanismes susceptibles de participer à cette altération des jeux épistémiques à des fins de formations initiales et continues de professeurs.

\section{Discussion}

\section{Les jeux alternatifs: dimensions génériques et méca- nismes liés à l'altération des jeux épistémiques}

Ce qui nous semble pouvoir être retenu des pratiques didactiques observées en géométrie et en escalade, c'est le primat accordé à la communication contractuelle au détriment du dialogue épistémique. Enseigner et apprendre consistent avant tout à procéder au maintien "coûte que coûte» d'une relation sociale.

Parallèlement, la dimension effective du milieu, en tant que système allié, au sens ou il doit poser le moins d'entrave possible à l'activité conjointe des acteurs, peut être également relevée comme une constante. La pratique ostensive des objets manipulés par le professeur et les élèves occupe la totalité de l'espace des échanges. De ce fait les enjeux épistémiques sous-jacents s'estompent au fur et à mesure de l'avancée du temps d'enseignement.

Il peut être également noté que les élèves ne perçoivent pas la dimension opératoire des savoirs. En d'autres termes, apprendre ne consiste pas, prioritairement, à construire des stratégies adaptatives aux situations, mais à assimiler des savoirs encapsulés à des situations pour satisfaire les demandes professorales. Ainsi, la logique discursive prévaut au détriment d'une logique praxique. 
Un quatrième élément nous semble contribuer à l'altération des enjeux épistémiques. Il s'agit de la dilution des responsabilités didactiques. Chacun agit comme si le savoir produit était de la seule responsabilité de l'autre. Corrélativement, le statut de l'erreur semble se limiter à un simple écart dû à une application non correcte de la règle, alors qu'elle est imputable à des stratégies inopérantes (Houdé, 2008).

Enfin, les élèves accordent un intérêt électif aux éléments appendices du contrat didactique, ceux qui apparaissent lors des actes de langage du professeur. Les pratiques didactiques de ces élèves en grandes difficultés semblent avant tout commandées par le souci de ne pas être perçus «hors du jeu» professoral.

En ce qui concerne les résultats produits, la caractérisation des formes contractuelles qui président à l'enchâssement des pratiques et de directions d'études mérite, à notre sens, d'être soulignée. Ces résultats recoupent ceux d'études antérieures en mathématiques (Joshua \& Dupin, 2003; Mercier, 2002), et en éducation physique et sportive (Amade-Escot, 2003) tout en les éclairant d'un jour nouveau.

Ils pointent notamment comment les processus mésogénétiques (alliés versus antagonistes) sont susceptibles de cristalliser les difficultés des élèves en 1) instaurant des rapports de surface aux objets de savoirs, 2) favorisant la dilution des responsabilités au fil des transactions, 3) rendant difficile en géométrie, voire impossible en escalade, la mise en mémoire de l'expérience.

Des résultats qui soulignent également que des élèves en grande difficulté scolaire développent des pratiques didactiques proches alors qu'ils sont confrontés à des enjeux de savoirs spécifiques.

Il est alors possible de présenter, sous la forme d'un tableau synoptique en guise de résumé, certaines des «règles» constitutives des jeux alternatifs.

\section{Conclusion}

La recherche menée se voulait avant tout exploratoire. Elle n'avait pas pour ambition de porter un regard sur les pratiques déployées par tous les élèves, pas plus qu'elle ne prétendait rendre compte des pratiques professorales de professeurs de mathématiques ou d'éducation physique et sportive.

Elle visait à questionner des pratiques d'élèves en grandes difficultés d'apprentissage appartenant à une même classe et confrontés à des enjeux de savoirs spécifiques. Il s'agissait de mettre en lumière, à partir d'une approche anthropologique en didactique, laquelle envisage l'activité d'un professeur et des élèves comme relevant d'une action conjointe (Sensevy, 2007), les stratégies développées par ces élèves, et de les comparer afin de tirer des enseignements utiles à la formation aux métiers de l'enseignement. 


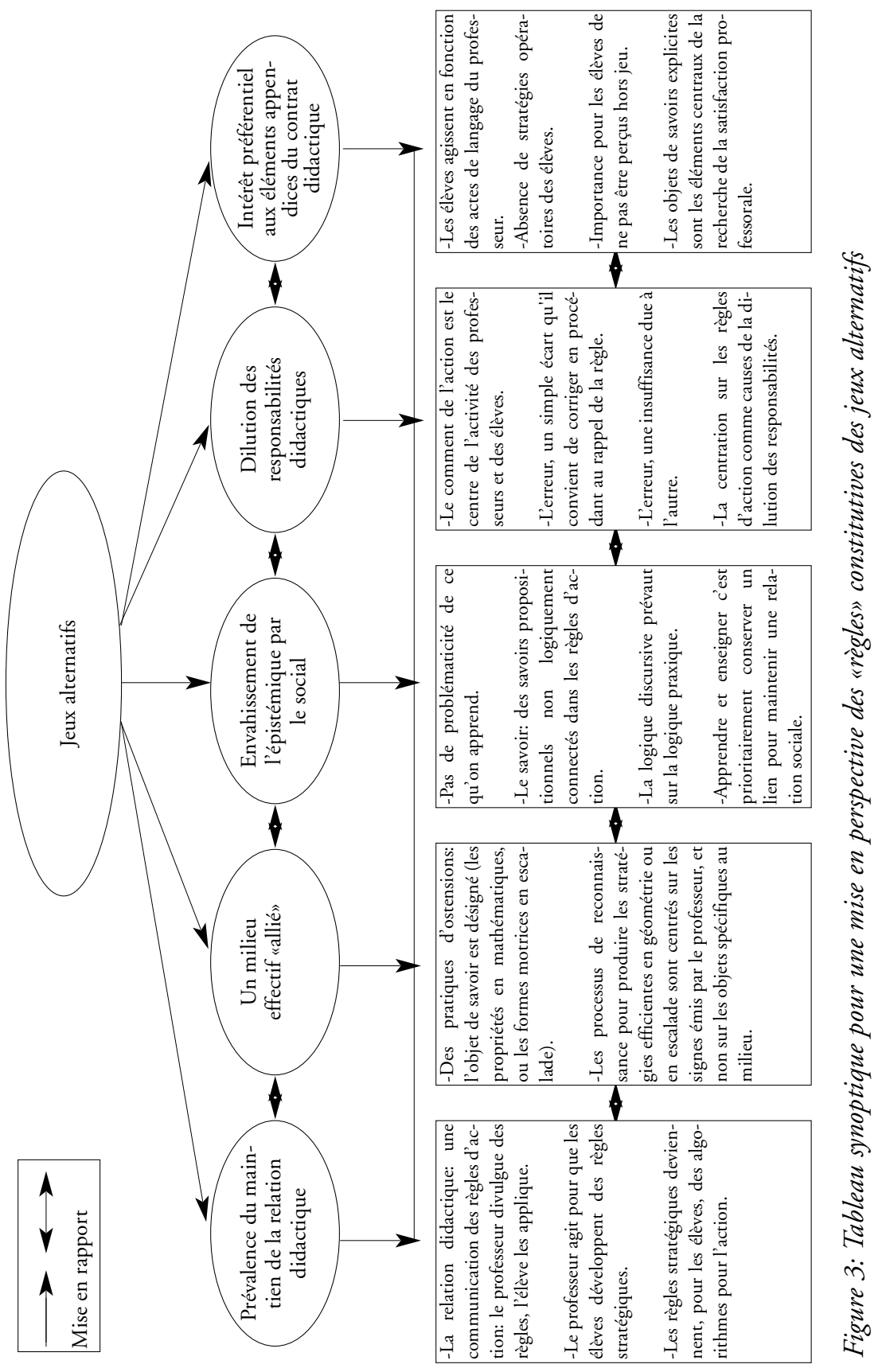


Nous avons ainsi pu mettre en lumière des phénomènes d'altération du jeu didactique, une altération dont l'origine doit être envisagée comme une co-construction professeurs-élèves. Si le professeur adopte une pratique d'enseignement, c'est avant tout pour répondre à l'urgence d'une situation problématique rencontrée par des élèves. Conjointement, l'activité didactique des élèves se limite à une forme de connaissances de nature déclarative.

Complémentairement, nous devons souligner que la construction de comparables suppose de ne pas se précipiter sur la quête d'une caractérisation générique aux dépens des spécificités. En effet, étant donné la nature des deux disciplines supports aux analyses des pratiques des élèves, la géométrie et l'escalade, nous sommes en présence de pratiques sociales de natures différentes et donc d'institutions très particulières à solliciter.

Il convient de ce fait d'opérer une insistance sur le spécifique, y compris les spécificités des positions des élèves, comme effets de mise en évidence de certaines dimensions génériques. C'est seulement après avoir mené une analyse spécifique des pratiques didactiques qu'il nous a été possible de mettre au jour certaines formes de pratiques alternatives génériques. Néanmoins, sans vouloir prétendre à l'exhaustivité, plusieurs points méritent d'être approfondis dans le cadre de recherches futures.

Ainsi la question des jeux d'apprentissages alternatifs, lorsqu'elle est associée à des pratiques didactiques non attendues par les professeurs au sein d'une population d'élèves déclarés en grandes difficultés, pourrait masquer l'intérêt de la notion pour penser les pratiques didactiques d'élèves dits «bons» et dont les jeux alternatifs correspondraient plutôt à des moyens pour éviter l'ennui d'une chronogénèse qui peine à avancer, ou encore la manifestation d'une connaissance privée dense du point de vue épistémique, mais dont le professeur ne parvient pas à saisir la logique ou la pertinence dans le flot des événements en cours. Cet aspect milite, plus que jamais, pour un prolongement de ce travail afin de mieux cerner les rapports évolutifs que peut entretenir un même élève avec les systèmes d'objets des deux disciplines.

\section{Notes}

1 Cette recherche a été menée dans le cadre d'une thèse intitulée «Étude didactique comparative de pratiques d'élèves au collège en Mathématiques et en Éducation Physique et Sportive: vers la notion de jeux alternatifs».

2 Cahier support de l'enseignement des mathématiques pour les classes de $4^{\mathrm{e}}$ de collège. Hatier, Paris. 1998).

3 Nous inscrivons cette approche dans le prolongement de la Théorie des Situations Didactiques (Brousseau, 1998) et de la Théorie Anthropologique du didactique (Chevallard, 1999). Nous nous situons dans une perspective actionnelle. Dans ce prolongement, les analyses menées portent sur les interactions langagières dans le cadre de pratiques ordinaires de classes

4 Le vertige vrai correspond à la sensation rotatoire et nauséeuse provoquée par une perception de mouvement différente entre la vision et l'organe vestibulaire. 
5 Les voies d'escalade étaient réparties sur une surface de 20 mètres de long. Leur hauteur était de 12 mètres. Notre présence, située à près de 10 mètres derrière les élèves et le professeur, ne semble pas avoir eu d'influence sur l'activité transactionnelle des acteurs.

6 Paraphraser consiste ici à identifier les sémantismes respectifs de séquences à partir d'un sujet agissant dans le cadre d'une situation aux enjeux de savoirs identifiés.

7 Altération est un concept de valeur qualitatif. Il semble donc particulièrement bien adapté aux pratiques didactiques relevées dans cette recherche.

8 Indique une partie inaudible.

9 Un niveau de prises correspond aux prises situées sur une même ligne horizontale.

\section{Bibliographie}

Amade-Escot, C. (2003). Didactique de l'éducation physique. État des recherches. Paris: Éditions de la revue éducation physique et sports, recherches et formation.

Austin, J. L. (1970). Quand dire c'est faire. Paris: Éditions du Seuil.

Bautier, E. \& Rayou, P. (2009). Les inégalités d'apprentissage. Paris: PUF.

Blanchard-Laville, C. (2002). Rapport au savoir: Que nous dit la clinique. Actes des troisièmes Journées Franco-Québécoises "Didactiques et rapports aux savoirs". Paris: La Sorbonne.

Bourdieu, P., Chamboredon, J.-C. \& Passeron, J.-C. (2005). Le métier de sociologue. Paris: Éditions de l'école des hautes études en sciences sociales.

Brousseau, G. (1998). Théorie des situations didactiques. Grenoble: La Pensée Sauvage.

Brousseau, G. (2003). Glossaire. Consulté le 20 janvier 2010 dans http://daest.pagespersoorange.fr/guybrousseau/textes/GlossaireBrousseau.pdf

Charaudeau, P. \& Maingueneau, D. (2002). Dictionnaire d'analyse du discours. Paris: Seuil.

Charlot, B. (1997). Du rapport au savoir. Paris: Anthropos.

Charlot, B. (2003). La problématisation du rapport au savoir. In S. Maury \& M. Caillot (Éd.), Rapport au savoir et didactiques (pp. 33-50). Paris: Fabert.

Chauvier, S. (2007). Qu'est-ce qu'un jeu. Paris: VRIN.

Chauviré, C. \& Sackur, C. (2003). Le vocabulaire de Wittgenstein. Paris: Ellipses.

Chevallard, Y. (1991). La transposition didactique du savoir savant au savoir enseigné. Grenoble: La Pensée Sauvage.

Chevallard, Y. (1999). Analyse des pratiques enseignantes et didactique des mathématiques (pp. 91-120). Clermont-Ferrand: IREM.

Deloustal-Jorrand, V. (2004). L’implication mathématique: Étude épistémologique et didactique. Thèse en Didactique des Mathématiques, Université Joseph Fourier, Grenoble.

Douglas, M. (1999). Comment pensent les institutions. Paris: La Découverte/M.A.U.S.S.

Do, C-L. (2007). Les dossiers. Les représentations de la grande difficulté scolaire par les enseignants. Année scolaire 2005-2006. 182 Ministère Education Mationale Enseignement Supérieure Recherche.

Durand, M., Saury, M. \& Sève, C. (2006). Apprentissage et configuration d'activité: Une dynamique ouverte des rapports sujets-environnements. In J.-M. Barbier \& M. Durand (Éd.), Sujets, activités, environnements (pp. 61-83). Paris: Presses Universitaires de France.

Durkheim, E. (2002). Les règles de la méthode sociologique. Paris: Presses Universitaires de France.

Duru-Bellat, M. \& Dubet, F. (2004). Qu'est-ce qu'une école juste? Revue Française de Pédagogie, 146, 105-114.

Forest, D. (2006). Analyse proxémique d'interactions didactiques. Thèse de doctorat en Sciences de l'éducation. Université Européenne de Bretagne, Rennes.

Ginzburg, C. (1989). Mythes, emblèmes, traces. Morphologie et histoire. Paris: Flammarion.

Houdé, O. (2008). Développement cognitif. In A. van Zanten (Éd.), Dictionnaire de l'éducation (pp. 126-129). Paris: Presses Universitaires de France. 
Hussenet, A. \& Santana, P. (2004). Le traitement de la grande difficulté scolaire au collège et à la fin de la scolarité obligatoire. (Rapport du Haut Conseil de l'évaluation de l'école No 13). Paris: MEN.

Joshua, S. \& Dupin, J.-J. (2003). Introduction à la didactique des sciences et des mathématiques. Paris: Presses Universitaires de France.

Lahire, B. (1998). L'homme pluriel. Paris: Éditions Nathan.

Laterasse, C. \& Brossais, E. (2006). Le Rapport au savoir. In J. Beillerot \& N. Mosconi (Éd.), Traité des sciences et des techniques de l'éducation (pp. 381-393). Paris: Dunod.

Mauss, M. (2001). Essai sur le don. In M. Mauss (Dir.), Sociologie et anthropologie. Paris: Presses universitaires de France.

Mercier, A. (2008). Pour une lecture anthropologique du «programme didactique». Éducation et didactique, Vol. 2, (1), 7-40.

Pascal, D. (1980). Le problème du zéro. L'économie de l'échec dans la classe et la production de l'erreur. Mémoire de diplôme d'études approfondies, Université d'Aix-Marseille II, Université de Bordeaux I.

Pastré, P. (2004). Introduction. Recherches en didactique professionnelle. In R. Samurçay \& P. Pastré (Éd.), Recherches en didactique professionnelle (pp. 1-13). Toulouse: Octares.

Rilhac, P. (2008). Étude didactique comparative de pratiques d'élèves au collège en mathématiques et en éducation physique et sportive: Vers la notion de jeux alternatifs. Thèse de doctorat inédite, Université Européenne de Bretagne, Rennes.

Schubauer-Leoni, M.-L. \& Leutenegger, F. (2002). Expliquer et comprendre dans une approche clinique/expérimentale du didactique ordinaire. In F. Leutenegger \& M. Saada-Robert (Éd.), Expliquer et comprendre en sciences de l'éducation (pp. 227-251). Bruxelles: De Boeck Université.

Schubauer-Leoni, M.-L., Leutenegger, F., Ligozat, F. \& Flückiger, A. (2007). Un modèle de l'action conjointe professeur-élèves: Les phénomènes didactiques qu'il peut/doit traiter. In G. Sensevy \& A. Mercier (Éd.), Agir ensemble (pp. 51-91). Presses Universitaires de Rennes.

Sensevy, G., Mercier, A., Schubauer-Leoni, M-L. (2000). Vers un modèle de l'action didactique du professeur. A propos de la course à 20. Recherches en didactiques des mathématiques, 20. (3), 263-304.

Sensevy, G., Mercier, A., Schubauer-Leoni, M-L., Ligozat, F. \& Perrot, G. (2005). An attempt to model the teacher's action in mathematics, Educational Studies in mathematics, 20. (3), 263-304.

Sensevy, G., Schubauer-Leoni, M.-L. \& Mercier, A. (2007). Pratiques d'éducation, vidéos, instruments. Un point de vue depuis la théorie de l'action conjointe en didactique. Actes du colloque VISA. Lyon.

Sensevy, G. (2007). Action conjointe professeur-élèves dans le système didactique. In G. Sensevy \& A. Mercier (Éd.), Agir ensemble (pp. 13-49). Presses Universitaires de Rennes.

Sensevy, G. (à paraître). Filmer la pratique. Un point de vue de la théorie de l'action conjointe en didactique. In L. Veillard \& A. Tiberghien (Éd.), Instrumentation de la recherche en Éducation. Le cas du développement d'une base de vidéos de situation d'enseignement et d'apprentissage VISA. Paris: Presses de la MSH.

Testevuide, S. (2003). L'escalade en situation. Paris: Éditions de la revue éducation physique et sports.

Vergnaud, G. \& Récopé, M. (2000). De Revault d'Allones à une théorie du schème aujourd'hui. Psychologie Française, 45, (1), 35-50.

Woods, P. (1990). L'ethnographie de l'école. Paris: Armand Colin.

Mots-clés: Pratiques didactiques, éducation physique et sportive, mathématiques, jeux alternatifs, enseignement secondaire obligatoire 


\section{Die Beziehung von Wissen und Anwendung bei Lernvor- gängen: Der Begriff des alternativen Spielens}

\section{Zusammenfassung}

Dieser Beitrag stellt die Ergebnisse einer Forschung vor, deren Ziel es war, die Lernstrategien von 13 Jahre alten Schülerinnen und Schülern zu vergleichen. Diese Schüler/innen sollten sich an einen spezifischen Lernkontext anpassen: In Mathematik wurde die mathematische Beweisführung und im Fach Sport das Klettern eingeführt. Der methodologische Ansatz, der dieser Arbeit zugrunde liegt, ist der Begriff des «methodologischen Algorithmus». Die Ergebnisse zeigen die Existenz «alternativer Spielarten», die so genannt werden, weil sie nicht genau den vom Lehrer erwarteten epistemologischen Spielarten entsprechen.

Schlagworte: Didaktische Praktiken, Sportunterricht, Mathematikunterricht, Wissen, alternative Spiele, Sekundarstufe 1

\section{La relazione tra sapere e applicazione nell'apprendimento: il caso dei giochi alternativi}

\section{Riassunto}

Questo contributo presenta i risultati di una ricerca che aveva come obiettivo il confronto delle strategie di apprendimento degli allievi di 13 anni. Questi allievi si devono adattare a un contesto di apprendimento specifico: in matematica vengono introdotte le dimostrazioni matematiche e l'arrampicata in educazione fisica. L'approccio metodologico proposto si fonda sul concetto di «algoritmo metodologico». I risultati mostrano l'esistenza di «modalità di gioco alternative», così chiamate in quanto non corrispondenti alle modalità di gioco epistemico attese dal docente.

Parole chiave: Pratiche didattiche, educazione fisica, matematica, giochi alternativo, scuola secondaria

\section{Knowledge relationship and pupils' practices: the notion of alternative games}

\section{Abstract}

This article presents the results of a study whose ambition is to compare students' relationship to knowledge when they are confronted with the following specific knowledge: demonstrating in mathematics, and climbing in physical education and sports. Our methodological approach relies on the notion of «methodological algorithm». Results show the existence of different forms of games 
we say alternative, because they are not the form of epistemic games expected by teachers.

Keywords: Didactical practices, physical and sport education, mathematics, alternative games, secondary compulsory education 